\title{
Implantability, Complications, and Follow-Up After Transjugular Intrahepatic Portosystemic Stent-Shunt Creation With the 6F Self-Expanding Sinus-SuperFlex-Visual Stent
}

\author{
Daniel Spira, ${ }^{1,}{ }^{*} J a k u b$ Wiskirchen, ${ }^{2}$ Ulrich Lauer, ${ }^{3}$ Dominik Ketelsen, ${ }^{4}$ Konstantin Nikolaou, ${ }^{4}$ and \\ Benjamin Wiesinger ${ }^{4}$ \\ ${ }^{1}$ Department of Diagnostic and Interventional Radiology, University Hospital Heidelberg, Heidelberg, Germany \\ ${ }^{2}$ Department of Radiology and Nuclear Medicine, Franziskus Hospital, Bielefeld, Germany \\ ${ }^{3}$ Department of Gastroenterology and Hepatology, Eberhard-Karls-University, Tubingen, Germany \\ ${ }^{4}$ Department of Diagnostic and Interventional Radiology, Eberhard-Karls-University, Tubingen, Germany \\ "Corresponding author: Daniel Spira, Department of Diagnostic and Interventional Radiology, University Hospital Heidelberg, Heidelberg, Germany. Tel: +49-6221566410, Fax: \\ +49-6221565730, E-mail: daniel_spira@yahoo.de
}

Received 2015 March 14; Revised 2015 May 14; Accepted 2015 June 08.

\begin{abstract}
Background: The transjugular intrahepatic portosystemic stent-shunt (TIPSS) builds a shortcut between the portal vein and a liver vein, and represents a sophisticated alternative to open surgery in the management of portal hypertension or its complications. Objectives: To describe clinical experiences with a low-profile nitinol stent system in TIPSS creation, and to assess primary and longterm success.

Patients and Methods: Twenty-six patients ( 5 females, 21 males; mean age 54.6 years) were treated using a low-profile $6 \mathrm{~F}$ selfexpanding sinus-SuperFlex-Visual stent system. The indication for TIPSS creation was refractory bleeding in 9 of the 26 patients, refractory ascites in 18 patients, and acute thrombosis of the portal vein confluence in one patient. Portosystemic pressure gradients before and after TIPSS, periprocedural and long-term complications, and the time to orthotopic liver transplantation (OLT) or death were recorded.

Results: The portosystemic pressure gradient was significantly reduced, from $20.9 \pm 6.3 \mathrm{mmHg}$ before to $8.2 \pm 2.3 \mathrm{mmHg}$ after TIPSS creation $(P<0.001)$. Procedure-related complications included acute tract occlusion $(n=2)$, liver hematoma $(n=1)$, hepatic encephalopathy $(n=1)$, and cardiac failure $(n=1)$. Three of the 26 patients had late-onset TIPSS occlusion (at 12,12, and 39 months after TIPSS creation). Three patients died within one week after the procedure due to their poor general condition (multiorgan failure, acute respiratory distress syndrome, necrotizing pancreatitis, and aspiration pneumonia). Another four patients succumbed to their underlying advanced liver disease within one year after TIPSS insertion. Seven patients underwent OLT at a mean time of 9.4 months after TIPSS creation.

Conclusion: The sinus-SuperFlex-Visual stent system can be safely deployed as a TIPSS device. The pressure gradient reduction was clinically sufficient to treat the patients' symptoms, and periprocedural complications were due to the TIPSS procedure per se rather than to the particular stent system employed in this study.
\end{abstract}

Keywords: TIPSS, Self-Expandable Stent, Low-Profile Stent System

\section{Background}

Since its first description by Richter et al. (1), the transjugular intrahepatic portosystemic stent-shunt (TIPSS) has advanced to become a standard procedure in interventional radiology. Today, it represents a sophisticated alternative to open surgery or endoscopy in the management of portal hypertension or its complications. The TIPSS shunting methodology, a shortcut between the portal vein and a liver vein, is comparable to a surgically created shunt, with the aim to lower the pressure gradient between the portal vein (PV) and the inferior vena cava (IVC). The major Achilles heel of this method during follow-up, however, is shunt reocclusion, resulting once again in a severe and harmful increase of the portal pressure (along with all other possible complications, such as variceal bleeding or refractory ascites). In the past decade, multiple studies were performed in order to optimize the procedure, as well as to identify the optimal material to be used for this type of intervention. Balloon-expandable stents, self-expanding stents, and polytetrafluoroethylene (PTFE)- 
covered stents have been used for TIPSS creation in order to reduce the reocclusion rate and to thus improve longterm patency (2-4). In PTFE-covered stents, the rates of patency, either short-term or at two years after TIPSS placement, were described as $87 \%$ and $77 \%$, respectively (5). Perarnau et al. (6) compared covered stents to bare-metal stents, and reported a higher two-year rate of shunt dysfunction for bare-metal stents (64\%) than for covered stents (44\%). Nevertheless, early post-TIPSS complications, risk of hepatic encephalopathy, and two-year survival did not differ between the groups. These data are in line with a study by Clark et al. (7), who concluded that while covered stents may improve patency, they do not mitigate post-shunt hepatic dysfunction and do not improve survival. Notably, the cost of PTFE-covered systems greatly exceeds the cost of bare-metal stent systems. Additionally, stent grafts require larger vascular sheaths and are more rigid, which makes them difficult to place in patients with very tortuous or angled vessels. Therefore, bare-metal stents must still be regarded as a valuable alternative to PTFE-covered stents.

\section{Objectives}

Here, we describe our experience using a low-profile 6F self-expanding nitinol stent system (sinus-SuperFlexVisual@, Optimed, Germany) with respect to implantability, visibility, and primary and long-term success (including patency rates and other parameters).

\section{Patients and Methods}

\subsection{Patient Population}

This retrospective study was approved by our institutional review board, which waived informed consent. Twenty-six patients were treated with a low-profile $6 \mathrm{~F}$ self-expanding sinus-SuperFlex-Visual@ stent system (Optimed, Ettlingen, Germany). All but three patients (who were intubated and considered to be high-priority emergency cases at the time of intervention) were informed about the procedure prior to the intervention, and gave written consent.

Five of the 26 patients were female (19\%) and 21 were male $(81 \%)$. The mean age of the patients was 54.6 years, with a range of $30-70$ years. In nine of the 26 patients, the indication for TIPSS creation was refractory bleeding from esophageal or gastric varices that could not be controlled endoscopically. In 18 patients, the indication for TIPSS creation was intractable ascites, and one patient had acute thrombosis of the confluence of the superior mesenteric and splenic veins due to necrotizing pancreatitis (with multiple possible causes). The majority of our patients (18 out of 26) suffered from liver cirrhosis due to excessive alcohol consumption, and five were afflicted with active viral hepatitis. Two patients had subacute Budd Chiari syndrome (one without any other risk factors and the other in combination with chronic hepatitis C). In three patients, the liver cirrhosis was cryptogenic.

\subsection{TIPSS Placement}

After ultrasound-guided puncture of the right internal jugular vein, a 0.035" Glidewire (Terumo, Eschborn, Germany) was positioned in the IVC. Then, after dilating the subcutaneous tissue with 5F and 7F dilators, a 45-cm-long 9F sheath (Cordis Endovascular, Langenfeld, Germany) was inserted. With the help of a 7F multi-purpose catheter or a 5F C2 Cobra catheter (Cordis Endovascular), the right hepatic vein $(n=23)$ or the left hepatic vein $(n=3)$ was catheterized. The sheath was advanced into the hepatic vein and a wedge portogram was obtained. Midazolam, meperidine (pethidine), and atropine were administered via venous access to achieve conscious sedation in the 23 patients who were not under general anesthesia. The portal vein was punctured under fluoroscopic control using $30^{\circ}$ or $60^{\circ}$ TIPSS needles (Optimed). Then, the guide wire was advanced into the portal system, and a C2 Cobra catheter ( $5 \mathrm{~F}$ if used for venous catheterization; otherwise $4 \mathrm{~F}$ ) was placed deep into the superior mesenteric vein. The guidewire was subsequently replaced with an Amplatz Superstiff Wire (Boston Scientific, Natick, MA, USA). The TIPSS tract was pre-dilated with a 5-mm angioplasty balloon (Fox, Abbott Vascular, Wetzlar, Germany), and one or two sinusSuperFlex-Visual stents (Optimed) were implanted under fluoroscopic control. The stent was dilated with an $8 \mathrm{~mm}$ or larger angioplasty balloon (Fox, Abbott Vascular) if the porto-venous gradient could not be reduced to $<12 \mathrm{mmHg}$. Pressure gradients between the PV and the IVC were assessed before dilatation and after establishing the shunt. If the sheath could not be advanced into the portal vein, an additional safety wire was placed into the liver vein, the tip of the sheath was placed into the junction of the liver veins, and the stent was positioned directly (Figure 1).

Pressure measurements were performed during the TIPSS procedure. After transjugular puncture of a portal vein branch, a $4 \mathrm{~F}$ multipurpose catheter was advanced into the portal vein, and pressure was measured within the portal vein and the right atrium via the vascular sheath, using a conventional pressure transducer (Medex). After TIPSS placement, pressure measurements were repeated. The porto-systemic pressure gradient was calculated as portal vein pressure minus right atrial pressure.

In five of the 26 patients, persistent relevant shunting despite successful TIPSS placement was observed, and esophageal or gastric varices were embolized during the 


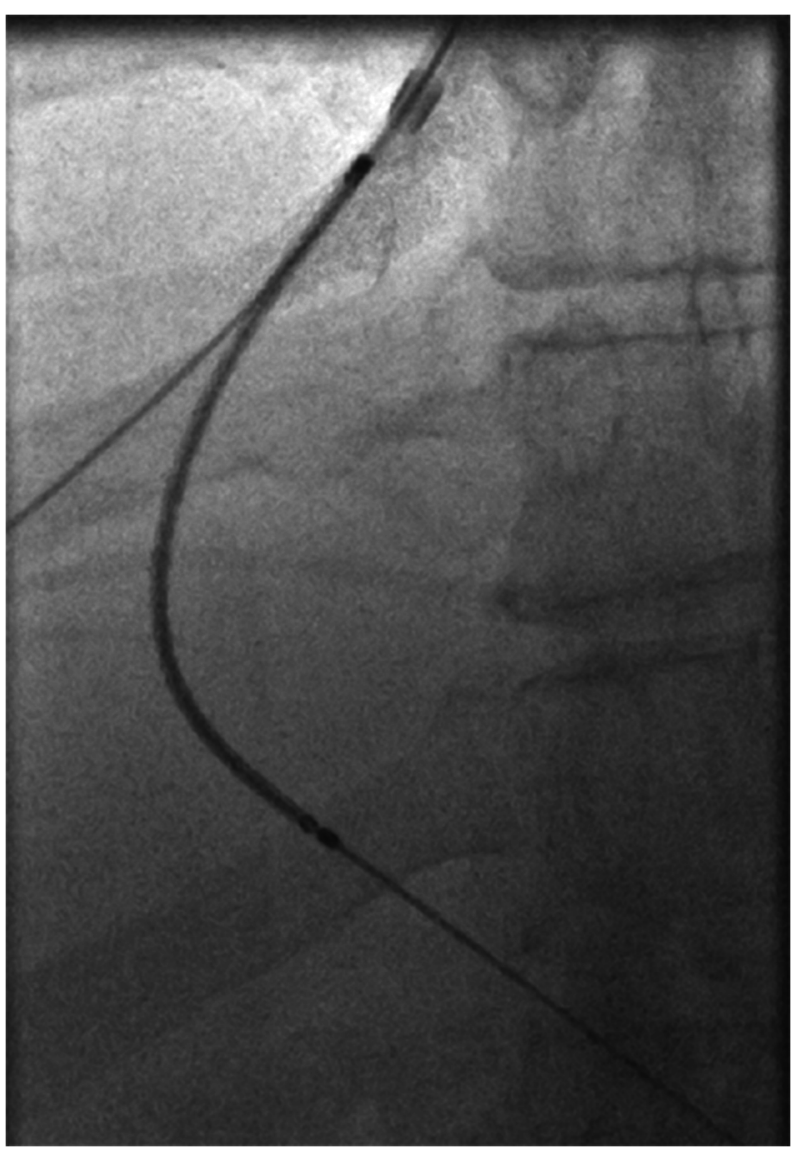

Figure 1. Sinus-SuperFlex-Visual stent in the liver before deployment; in this patient, advancing the sheath to the portal vein was not possible for technical reasons, so a stabilizing wire was placed in the right liver vein; using an Amplatz guidewire, the stent was positioned within the pre-dilated tract in the liver; when using a $9 \mathrm{~F}$ sheath, the stent can be easily positioned parallel to a second stabilizing wire.

same session. In four patients, coils (Cook Medical, Bloomington, IN, USA) measuring up to a diameter of $15 \mathrm{~mm}$ were used, and in one patient, an amplatzer plug (AGA Medical, Plymouth, MA, USA) was inserted to occlude a large gastric vein. In another four patients, who presented with refractory bleeding, a reduction of the porto-venous pressure gradient to $<8 \mathrm{mmHg}$ precluded relevant shunting and was sufficient to prevent re-bleeding.

\subsection{Follow-Up}

All patients received a control ultrasound within the first 48 hours after TIPSS creation in order to rule out acute shunt occlusion. Over the following month, the patients underwent Doppler sonography to control for TIPSS patency, and had physical examinations at internal medicine outpatient clinics to monitor for clinical signs of progressive liver disease. In 19 of the 26 patients, follow-up for $\geq$
5 years was documented. Seven of the 26 patients escaped long-term follow-up due to a change of residence or referral back to their home hospital after successful TIPSS placement.

TIPSS patency is of prime importance to reduce the risk of bleeding. Due to its short half-life, unfractionated heparin can be easily antagonized in cases of re-bleeding. Hence, immediately after TIPSS placement, every patient was placed on unfractionated heparin for at least 24 hours. Full heparinization was performed with heparin 25000 IU/24 hours, for an attempted partial thromboplastin time of 55 - 70 seconds. However, one patient developed a large capsular liver hematoma. With respect to that patient, we later abandoned the application of full heparinization after TIPSS.

\subsection{Statistical Analysis}

Data are reported as the arithmetic mean \pm standard deviation (and range). Statistical analysis was performed with the paired t-test. P value of $\leq 0.05$ were considered statistically significant.

\section{Results}

\subsection{TIPSS Creation}

TIPSS creation with a sinus-SuperFlex-Visual selfexpandable stent was successful in all patients. In 10 patients, a single stent with a nominal diameter of $12 \mathrm{~mm}$ and a length of $60 \mathrm{~mm}$ was used; in 13 patients, the stent length was $80 \mathrm{~mm}$; and in three patients, two stents had to be implanted (two with $12 / 60 \mathrm{~mm}$ plus $12 / 80 \mathrm{~mm}$ sinus stents, and the other with a $12 / 80 \mathrm{~mm}$ sinus stent plus a balloon-expandable stent). In 24 of the 26 patients, stents were deployed exactly at the pre-planned site (92\%), while in the remaining two patients, the stent was placed too deeply into the portal vein, so that an additional sinus stent (12/80 mm plus $12 / 60 \mathrm{~mm}$ ) had to be placed. In one patient, even though the stent length was sufficient, an additional balloon-expandable stent (Palmaz Genesis, Cordis Endovascular, Langenfeld, Germany) had to be inserted in order to achieve tract-straightening. No problems occurred with the pull-back deployment mechanisms.

The mean pressure gradient prior to the intervention was $20.9 \pm 6.3 \mathrm{mmHg}$ (range: 12 - $32 \mathrm{mmHg}$ ). Directly after the intervention, the pressure gradient dropped significantly to a mean of $8.2 \pm 2.3 \mathrm{mmHg}$ (range: $3-13)(\mathrm{P}<$ 0.001). In 19 of 26 patients, dilating the stent with an $8-\mathrm{mm}$ balloon was sufficient to lower the pressure gradient to $\leq$ $12 \mathrm{mmHg}$ (Figure 2A and B). In five patients, the stent was dilated to $10 \mathrm{~mm}$, and in one patient, the stent had to be dilated to $12 \mathrm{~mm}$ to sufficiently lower the pressure gradient. 
In one other patient, the stent was dilated to only $5 \mathrm{~mm}$, as the indication for the procedure was to coil existing varices rather than to create a relevant shunt, due to preexisting hepatic encephalopathy. In this patient, the portosystemic pressure gradient was left at $13 \mathrm{mmHg}$. In four of the $26 \mathrm{pa}-$ tients, additional coil embolization of esophageal and/or gastric varices was performed, and in one patient, an amplatzer plug was used to occlude a large variceal vein (Figure $3 \mathrm{~A}-\mathrm{C}$ ). One patient presented with acute thrombosis of the confluence of the superior mesenteric and splenic veins due to necrotizing pancreatitis. TIPSS insertion was successful, and the thrombotic material in the superior mesenteric and splenic veins was defragmented, leading to improved mesenteric venous flow.

\subsection{Periprocedural Complications}

We observed procedure-related complications in five patients, including acute tract occlusion in two patients, liver hematoma in one, hepatic encephalopathy in one, and cardiac failure in another. One patient, who received a $12 / 80 \mathrm{~mm}$ stent for the treatment of subacute BuddChiari syndrome, suffered from an acute stent occlusion within the first 24 hours post-intervention. The tract had to be recanalized, and two additional stents (Palmaz Genesis 9/59 and SMART 12/80, Cordis Endovascular, Langenfeld, Germany) were implanted. No occlusions were subsequently observed. A second patient, who was treated for refractory ascites, suffered from an acute TIPSS occlusion one day after TIPSS creation. In this patient, a VIATORR stent graft (W.L. Gore, Flagstaff, AZ, USA) was implanted, and no occlusion subsequently occurred. A third patient, who was being treated for refractory ascites and was suffering from cirrhosis of his already-transplanted liver, developed a large liver hematoma due to excessive post-interventional anticoagulation with heparin (PTT > 160). In the course of this intrahepatic bleeding, the patient developed transient renal insufficiency. Over the following six months, renal function recovered and the ascites was effectively controlled. This patient later underwent OLT. A fourth patient, suffering from cirrhosis due to chronic hepatitis $\mathrm{C}$, drug abuse, and excessive alcohol consumption, presented with a pre-interventional pressure gradient of $30 \mathrm{mmHg}$. Post-intervention, the pressure gradient was found to be lowered to $7 \mathrm{mmHg}$. This patient subsequently developed severe hepatic encephalopathy, which was successfully controlled with conservative treatment. A fifth patient developed cardiac insufficiency six months after TIPSS creation. The TIPSS tract was reduced by using a reduction stent (Optimed). Two days after shunt reduction, this patient underwent OLT.

\subsection{Follow-Up}

Three of the 26 treated patients (11.5\%) died within one week after the procedure. One was due to multiorgan failure, the second was due to acute respiratory distress syndrome that complicated preexisting necrotizing pancreatitis, and the third patient died due to pulmonary exhaustion resulting from pneumonia caused by aspiration.

Another four of the 26 patients (15.4\%) died within one year after TIPSS creation. In one case, ascites production was found to be significantly reduced after TIPSS insertion; however, this patient developed a continuously aggravating wasting syndrome and died nine months later. Another patient's status progressively deteriorated due to persistent alcohol abuse and refractory ascites. In the end, he denied medical support and succumbed to his progressive liver failure at six months. Another patient died with worsening ascites and hepatic encephalopathy at five months post-intervention. One patient, who received TIPSS because of uncontrollable bleeding from gastric varices, succumbed to re-bleeding.

Seven of the 26 patients underwent OLT at a mean time of 9.4 months (range: two days to 16 months) postintervention. One of these patients had late-onset TIPSS occlusion at 12 months after the TIPSS creation, which became symptomatic with re-bleeding of gastric varices. The TIPSS was recanalized and the patient received OLT one month later. In the remainder of these cases, the TIPSS tracts were consistently patent until the moment of OLT (Figure 4).

Two other patients presented with late-onset TIPSS occlusions. One became symptomatic 12 months after TIPSS placement, with re-bleeding from gastric varices. The other remained in good general condition, without varicose bleeding or refractory ascites, and the TIPSS occlusion was noticed incidentally during a routine duplexultrasound undertaken 39 months after TIPSS creation. As this patient was completely asymptomatic, no recanalization of the TIPSS was performed.

\section{Discussion}

TIPSS is the treatment of choice to manage the complications of portal hypertension, such as variceal hemorrhage or refractory ascites, when conservative or endoscopic procedures have failed. TIPSS creation significantly reduces the risk of bleeding from esophageal or gastric varices $(8,9)$, and is superior to conservative therapy in treating refractory ascites (10). However, establishing a TIPSS is associated with a significantly higher risk of hepatic encephalopathy $(8,10,11)$, as blood from the GI tract bypasses the liver and detoxification is significantly reduced. 

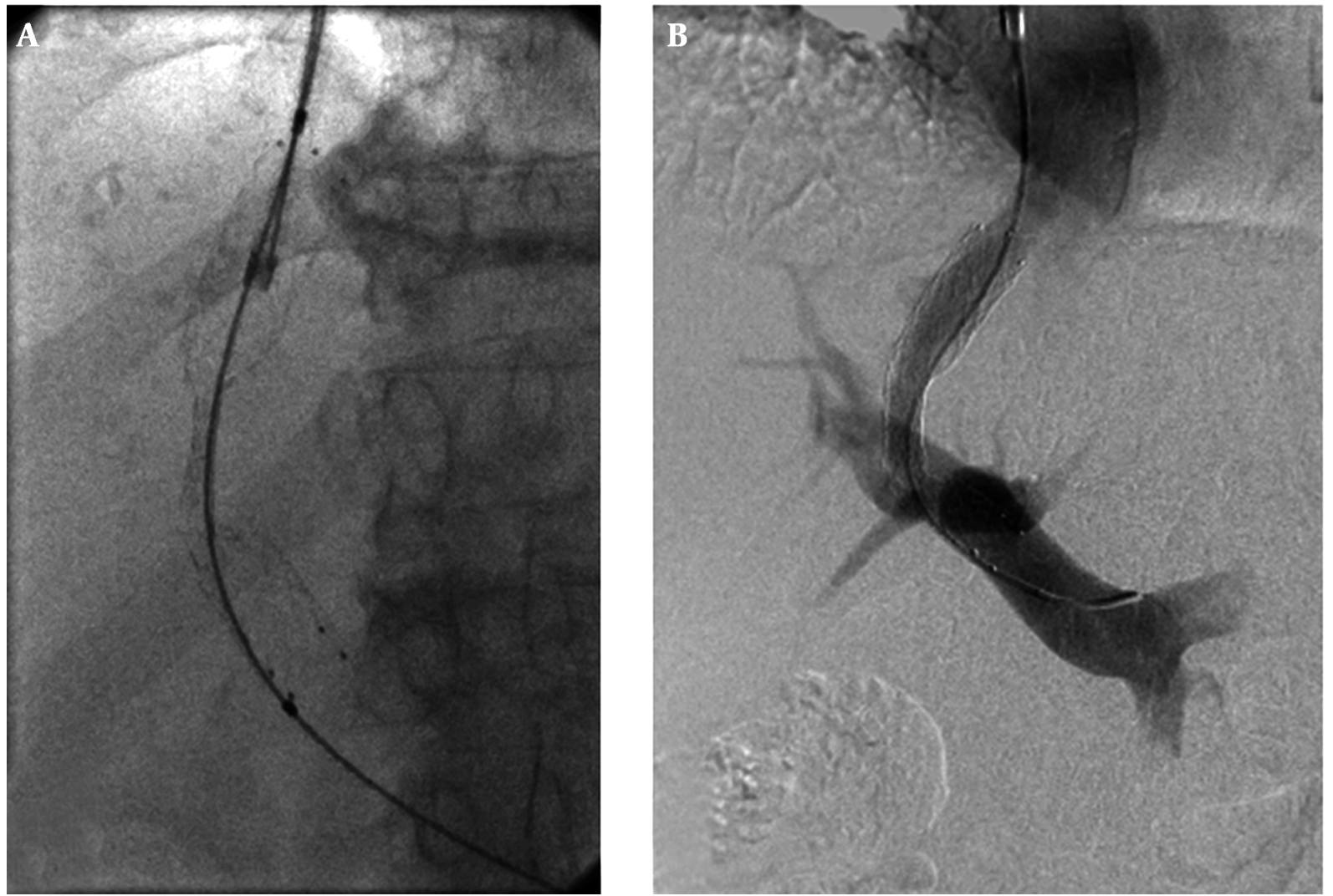

Figure 2. Sinus-SuperFlex-Visual stent (12/80 mm) after deployment; A, Prior to dilatation; B, The stent (12/80 mm) is dilated to $8 \mathrm{~mm}$; the portosystemic pressure gradient has been reduced to $5 \mathrm{mmHg}$ post-intervention, and the stent is not dilated further.
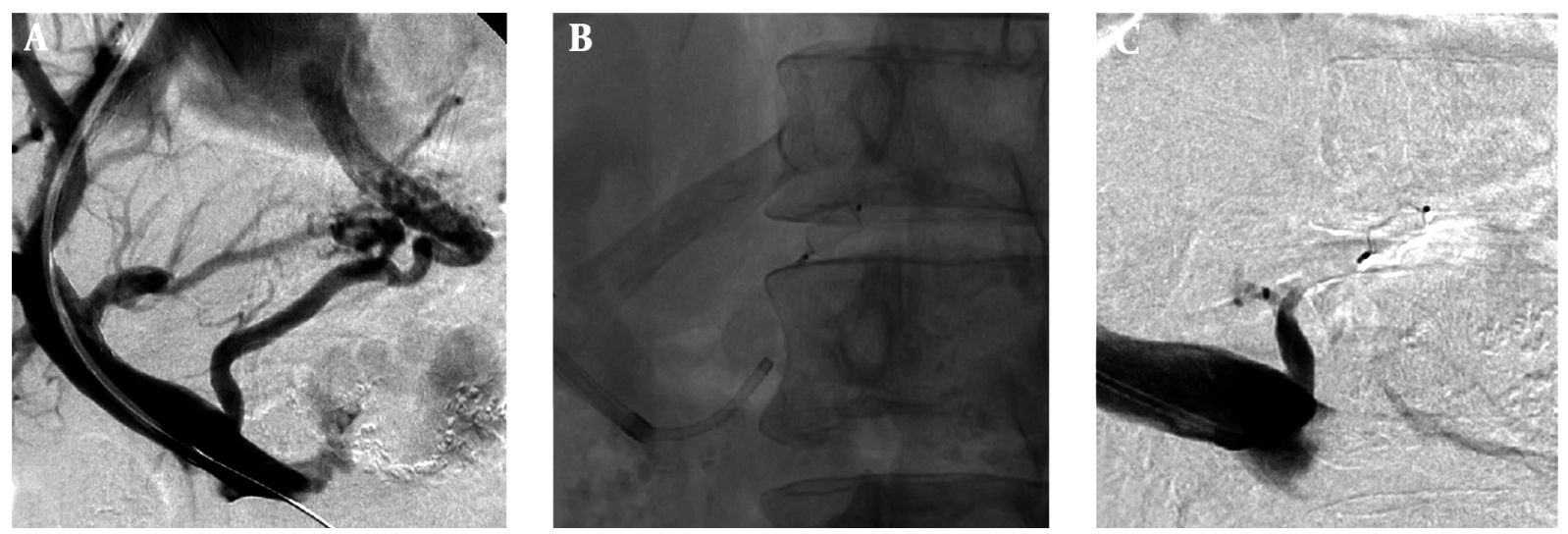

Figure 3. Patient suffering from recurrent variceal bleeding; A, After creating the portosystemic shunt, varices are still opacified; B, An amplatzer plug device is deployed in the major variceal vein, note the typical dog-bone configuration as an implication of optimal sizing; $\mathrm{C}$, Five minutes after deploying the amplatzer plug, the variceal vein no longer opacifies.

Nevertheless, hepatic encephalopathy following TIPSS creation can be managed conservatively in the majority of cases.
The technical success rate of establishing a proper shunt between the PV and the IVC has risen to approximately $95 \%$ over the years, due to the development of 


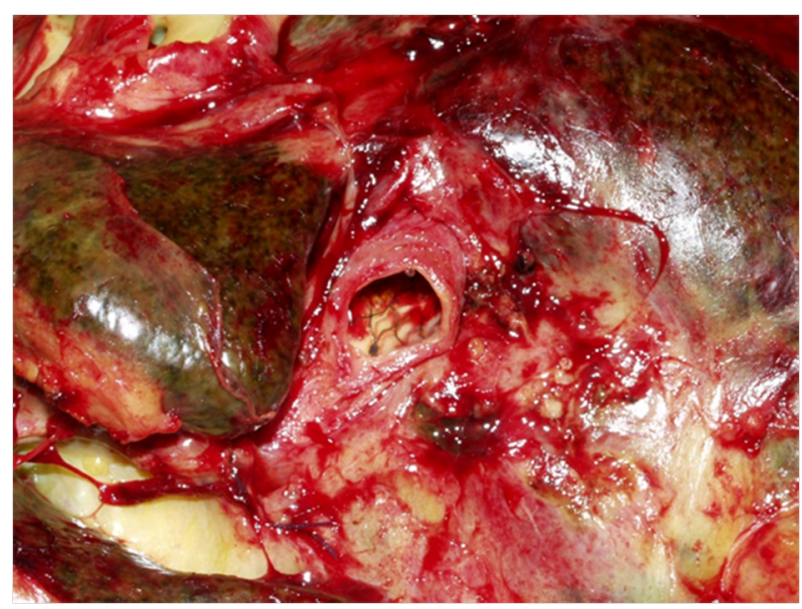

Figure 4. Sinus-SuperFlex-Visual stent in situ; the patient underwent OLT 15 months after TIPSS insertion. At the time of surgery, the TIPSS was still patent.

sophisticated tools, including low-profile balloons and stents $(12,13)$. Meanwhile, several stents or stent grafts have been evaluated for TIPSS creation. In the early days, stents such as balloon-expandable Palmaz stents were used with technical success rates ranging between $75 \%$ and $95 \%$ (1416). Subsequently, self-expanding stents such as the Wallstent have been evaluated $(16,17)$, and more recently, stent grafts such as the VIATORR have been used $(18,19)$. Compared to bare-metal stents, the long-term patency rates of stent grafts are higher, as no pseudointimal hyperplasia occludes the tract lumen. However, those grafts require larger vascular sheaths and, due to their rigidity, they can be difficult to place in patients with very tortuous or angled vessels.

In this paper, we describe our experience with a $6 \mathrm{~F}$ selfexpanding nitinol stent system. In order to enhance its visibility, radiopaque tantalum markers are fixed to the stent at both ends, which can be helpful during abdominal interventions, especially in obese patients or when using a flat-panel (digital) angiography unit (20). Due to its low profile and flexibility, this stent system can be introduced even if the transhepatic tract is very sloped. Furthermore, this system can be advanced through the pre-dilated liver parenchyma without the sheath having to be placed within the portal vein, making TIPSS establishment much easier, at least in some cases. When using, for example, a 9F vascular sheath, a second wire can be placed through the sheath for anchoring and stabilizing the vascular sheath in the liver vein (Figure 1). This is advantageous when the angle between the liver veins and the IVC is close to $90^{\circ}$, or when the IVC is found to be dilated.

We exclusively chose stents with a diameter of $12 \mathrm{~mm}$, to have the option of creating single shunts with a large diameter in order to avoid the implantation of a second, parallel shunt (21). In one patient, it was necessary to dilate the stent with a $12 \mathrm{~mm}$ balloon in order to sufficiently lower the pressure gradient. In 25 of 26 patients, we were able to reduce the portosystemic pressure gradient to $\leq$ $12 \mathrm{mmHg}$, which is considered the therapeutic goal, at the time of TIPSS creation $(22,23)$. Thus, the low-profile sinusSuperFlex-Visual stent was demonstrated to be powerful enough to achieve the therapeutically required pressure reduction. In one patient, we did not dilate the tract after stent placement, as our specific goal in this case was merely to embolize the variceal veins, which were the source of repetitive bleeding. We did not dilate the tract, due to preexisting hepatic encephalopathy, and we did not choose an abdominal percutaneous approach for coil embolization, due to ascites.

Additional embolization of variceal (esophageal and/or gastric) veins was performed in five of our 26 patients. In those five patients, the variceal veins still presented with the relevant shunting, although a TIPSS was created successfully and the portosystemic pressure had been lowered significantly, to below the threshold of $12 \mathrm{mmHg}$. As the indication for TIPSS creation in these patients was recurrent bleeding, we intended to lower the risk of re-bleeding by performing this additional procedure (24). In four of these five patients, coil embolization was performed, and in one patient, an amplatzer plug was inserted (Figure $3 \mathrm{~A}-\mathrm{C}$ ). In all patients, the varices no longer opacified after the intervention.

We observed relevant periprocedural complications in five of our 26 patients that were procedure-related rather than stent-related. One liver hematoma was caused in the course of the multiple punctures required to establish portal venous access, and by a prolonged PTT of $>$ 160 seconds due to unintentional excessive administration of heparin. In another patient, stent occlusion occurred within 24 hours after TIPSS creation. This early stent occlusion was likely due to the stent length chosen for the procedure; the stent did not completely cover the liver vein, which has been described as a reason for TIPSS failure by other groups $(4,16)$. A re-intervention with the implantation of two additional stents was necessary, and no further adverse events were noted thereafter in this patient. Another patient with acute tract occlusion was treated with the additional implantation of a VIATORR stent. A patient with hepatic encephalopathy was managed by a change in diet. One patient with cardiac decompensation six months after TIPSS creation was treated using a TIPSS reduction stent system. Two days later, this patient underwent OLT, and subsequently performed well. Overall, three patients died within one week after TIPSS creation, 
attributable to their limited general conditions (multiorgan failure, ARDS, pneumonia) rather than to the TIPSS creation. By and large, the rate of adverse effects was concordant with the rate of TIPSS-related complications already published in the literature $(25,26)$. Apparently, those side effects were procedure-related rather than graft-related.

With regard to long-term follow-up, four of the 26 patients in this study succumbed to their underlying advanced liver disease within one year after TIPSS insertion. In addition, three patients exhibited late-onset TIPSS occlusions (at 12,12, and 39 months after TIPSS creation). Overall, the long-term disease courses and late complications were found to be comparable to those reported by other groups $(6,7)$. Therefore, the TIPSS procedure is regarded as a highly effective bridge to liver transplantation, especially in patients with complications resulting from severe portal hypertension, and who are candidates for OLT. Indeed, seven of the 26 patients in this study underwent OLT at a mean time of 9.4 months after TIPSS creation. The shunt did not impair liver transplantation in any of these patients, which is in line with the results obtained in other studies $(27,28)$.

There are several limitations to our analysis that should be discussed. First, the data presented in this paper were analyzed retrospectively. Initially, our intention was to "only" treat the patients, not to evaluate or compare the function of different stent devices. Thus, there was no matched control group. Prospective, randomized trials focusing on patient survival are needed in order to compare the sinus-SuperFlex-Visual stent to other stent systems, and to avoid bias due to incomplete follow-up and retrospective analysis. Second, seven of the 26 patients escaped longterm follow-up due to a change of residence or referral back to their home hospital after successful TIPSS placement. Third, although the majority of our TIPSS patients most likely resembled each other with respect to their underlying conditions (i.e. alcoholism, viral hepatitis, cryptogenic cirrhosis, etc.), we cannot rule out that the stent system described in our analysis might be less beneficial in special subgroups of patients.

In conclusion, the sinus-SuperFlex-Visual stent system was found to be easily deployed even in the presence of disadvantageous vascular anatomy. The radiopaque markers made the stent well-visualized in all patients. The achieved rate of pressure gradient reduction (from a mean of 20.9 $\mathrm{mmHg}$ to a mean of $8.2 \mathrm{mmHg}$ ) was clinically sufficient to treat symptoms resulting from portal hypertension, and the rate of adverse effects was found to be similar to that of previously published data.

\section{Footnotes}

Authors' Contribution: All authors contributed substantially to the writing of the manuscript and approved its final submitted version.

Financial Disclosure: We herewith declare no conflicts of interest or financial disclosures for the authors of this manuscript.

Funding/Support: This study has not been sponsored.

\section{References}

1. Richter GM, Noeldge G, Palmaz JC, Roessle M. The transjugular intrahepatic portosystemic stent-shunt (TIPSS): results of a pilot study. Cardiovasc Intervent Radiol. 1990;13(3):200-7. [PubMed: 2121348].

2. Kuhn-Fulton J, Trerotola SO, Harris VJ, Snidow JJ, Johnson MS, Carey MA, et al. Transjugular intrahepatic portosystemic shunt procedure: efficacy of 10-mm versus 12-mm Wallstents. Radiology. 1996;199(3):658-64. doi: 10.1148/radiology.199.3.8637983. [PubMed: 8637983].

3. ter Borg PC, Hollemans M, Van Buuren HR, Vleggaar FP, Groeneweg M, Hop WC, et al. Transjugular intrahepatic portosystemic shunts: longterm patency and clinical results in a patient cohort observed for 39 years. Radiology. 2004;231(2):537-45. doi: 10.1148/radiol.2312021797. [PubMed: 15044746].

4. Cejna M, Peck-Radosavljevic M, Thurnher SA, Hittmair K, Schoder $\mathrm{M}$, Lammer J. Creation of transjugular intrahepatic portosystemic shunts with stent-grafts: initial experiences with a polytetrafluoroethylene-covered nitinol endoprosthesis. Radiology. 2001;221(2):437-46. doi: 10.1148/radiol.2212010195. [PubMed: 11687688].

5. Sajja KC, Dolmatch BL, Rockey DC. Long-term follow-up of TIPS created with expanded poly-tetrafluoroethylene covered stents. Dig Dis Sci. 2013;58(7):2100-6. doi: 10.1007/s10620-013-2578-0. [PubMed: 23381105].

6. Perarnau JM, Le Gouge A, Nicolas C, d'Alteroche L, Borentain P, Saliba F, et al. Covered vs. uncovered stents for transjugular intrahepatic portosystemic shunt: a randomized controlled trial. J Hepatol. 2014;60(5):962-8. doi: 10.1016/j.jhep.2014.01.015. [PubMed: 24480619].

7. Clark W, Golkar F, Luberice K, Toomey P, Paul H, Marcadis A, et al. Uncovering the truth about covered stents: is there a difference between covered versus uncovered stents with transjugular intrahepatic portosystemic shunts?. Am J Surg. 2011;202(5):561-4. doi: 10.1016/j.amjsurg.2011.06.021. [PubMed: 21944293].

8. Luca A, D’Amico G, La Galla R, Midiri M, Morabito A, Pagliaro L. TIPS for prevention of recurrent bleeding in patients with cirrhosis: metaanalysis of randomized clinical trials. Radiology. 1999;212(2):411-21. doi: 10.1148/radiology.212.2.r99au46411. [PubMed: 10429698].

9. Tripathi D, Therapondos G, Jackson E, Redhead DN, Hayes PC. The role of the transjugular intrahepatic portosystemic stent shunt (TIPSS) in the management of bleeding gastric varices: clinical and haemodynamic correlations. Gut. 2002;51(2):270-4. [PubMed: 12117893].

10. Deltenre P, Mathurin P, Dharancy S, Moreau R, Bulois P, Henrion J, et al. Transjugular intrahepatic portosystemic shunt in refractory ascites: a meta-analysis. Liver Int. 2005;25(2):349-56. doi: 10.1111/j.14783231.2005.01095.x. [PubMed: 15780061].

11. Somberg KA, Riegler JL, LaBerge JM, Doherty-Simor MM, Bachetti P, Roberts JP, et al. Hepatic encephalopathy after transjugular intrahepatic portosystemic shunts: incidence and risk factors. Am J Gastroenterol. 1995;90(4):549-55. [PubMed: 7717309].

12. Charon JP, Alaeddin FH, Pimpalwar SA, Fay DM, Olliff SP, Jackson $\mathrm{RW}$, et al. Results of a retrospective multicenter trial of the Viatorr expanded polytetrafluoroethylene-covered stent-graft for tran- 
sjugular intrahepatic portosystemic shunt creation. J Vasc Interv Radiol. 2004;15(11):1219-30. doi: 10.1097/01.RVI.0000137434.19522.E5. [PubMed: 15525740].

13. Schaefer PJ, Jahnke T, Schaefer FK, Hedderich J, Hinrichsen H, Heller $\mathrm{M}$, et al. [Transjugular intrahepatic portosystemic shunt: evaluation of the impact of the stent's configuration on the patency rate]. Rofo. 2007;179(9):965-70. doi: 10.1055/s-2007-963394. [PubMed: 17705118].

14. Noeldge G, Richter GM, Roessle M, Haag K, Katzen BT, Becker GJ, et al. Morphologic and clinical results of the transjugular intrahepatic portosystemic stent-shunt (TIPSS). Cardiovasc Intervent Radiol. 1992;15(5):342-8. [PubMed: 1423396].

15. Richter GM, Roeren T, Brado M, Noldge G, Kauffmann GW. [Long-term results after TIPSS with the Palmaz stent]. Radiologe. 1994;34(4):17882. [PubMed: 8052709].

16. Tesdal IK, Jaschke W, Buhler M, Adamus R, Filser T, Holm E, et al. Transjugular intrahepatic portosystemic shunting (TIPS) with balloonexpandable and self-expanding stents: technical and clinical aspects after 31/2 years' experience. Cardiovasc Intervent Radiol. 1997;20(1):2937. [PubMed: 8994721].

17. Borsa JJ, Fontaine AB, Hoffer EK, Bloch RD, Tong E, Kuhr CS, et al. Retrospective comparison of the patency of Wallstents and Palmaz longmedium stents used for TIPS. Transjugular intrahepatic portosystemic shunts. Cardiovasc Intervent Radiol. 2000;23(5):332-9. [PubMed: 11060361].

18. Hausegger KA, Karnel F, Georgieva B, Tauss J, Portugaller H, Deutschmann $\mathrm{H}$, et al. Transjugular intrahepatic portosystemic shunt creation with the Viatorr expanded polytetrafluoroethylenecovered stent-graft. J Vasc Interv Radiol. 2004;15(3):239-48. [PubMed: 15028808].

19. Rossi P, Salvatori FM, Fanelli F, Bezzi M, Rossi M, Marcelli G, et al. Polytetrafluoroethylene-covered nitinol stent-graft for transjugular intrahepatic portosystemic shunt creation: 3-year experience. Radiology. 2004;231(3):820-30. doi: 10.1148/radiol.2313030349. [PubMed: 15118117].

20. Wiesinger B, Stutz A, Schmehl J, Claussen CD, Wiskirchen J. Comparison of digital flat-panel detector and conventional angiography machines: evaluation of stent detection rates, visibility scores, and dose-area products. AJR Am J Roentgenol. 2012;198(4):946-54. doi 10.2214/AJR.11.7245. [PubMed: 22451565].

21. He FL, Wang L, Yue ZD, Zhao HW, Liu FQ. Parallel transjugular intrahepatic portosystemic shunt for controlling portal hypertension complications in cirrhotic patients. World J Gastroenterol. 2014;20(33):118359. doi: 10.3748/wjg.v20.i33.11835. [PubMed: 25206289].

22. Sanyal AJ, Freedman AM, Luketic VA, Purdum PP., Shiffman ML, DeMeo J, et al. The natural history of portal hypertension after transjugular intrahepatic portosystemic shunts. Gastroenterology. 1997;112(3):88998. [PubMed: 9041251].

23. Sahagun G, Benner KG, Saxon R, Barton RE, Rabkin J, Keller FS, et al. Outcome of 100 patients after transjugular intrahepatic portosystemic shunt for variceal hemorrhage. Am J Gastroenterol. 1997;92(9):1444-52. [PubMed: 9317060].

24. Tesdal IK, Filser T, Weiss C, Holm E, Dueber C, Jaschke W. Transjugular intrahepatic portosystemic shunts: adjunctive embolotherapy of gastroesophageal collateral vessels in the prevention of variceal rebleeding. Radiology. 2005;236(1):360-7. doi: 10.1148/radiol.2361040530. [PubMed: 15955858].

25. Casadaban LC, Parvinian A, Minocha J, Lakhoo J, Grant CW, Ray CEJr., et al. Clearing the Confusion over Hepatic Encephalopathy After TIPS Creation: Incidence, Prognostic Factors, and Clinical Outcomes. Dig Dis Sci. 2015;60(4):1059-66. doi: 10.1007/s10620-014-3391-0. [PubMed: 25316553].

26. Qin JP, Jiang MD, Tang W, Wu XL, Yao X, Zeng WZ, et al. Clinical effects and complications of TIPS for portal hypertension due to cirrhosis: a single center. World J Gastroenterol. 2013;19(44):8085-92. doi: 10.3748/wjg.v19.i44.8085. [PubMed: 24307804].

27. Barbier L, Hardwigsen J, Borentain P, Biance N, Daghfous A, Louis G et al. Impact of transjugular intrahepatic portosystemic shunting on liver transplantation: 12-year single-center experience. Clin Res Hepatol Gastroenterol. 2014;38(2):155-63. doi: 10.1016/j.clinre.2013.09.003. [PubMed: 24183545].

28. Saad WE. Transjugular Intrahepatic Portosystemic Shunt before and after Liver Transplantation. Semin Intervent Radiol. 2014;31(3):243-7. doi: 10.1055/s-0034-1382791. [PubMed: 25177084]. 